\title{
ANÁLISIS ECOLÓGICO BÁSICO DE LOS GASTRÓPODOS TERRESTRES DE LA REGIÓN ORIENTAL DE SAN LUIS POTOSÍ, MÉXICO
}

\author{
Alfonso Correa-SAndoval', Ned E. STRenth ${ }^{2}$, Rubén Rodríguez CaSTro1 \\ y Jorge Víctor HoRTA VEGA \\ ${ }^{1}$ Departamento de Biología, Instituto Tecnológico de Cd. Victoria, Tams., Apdo. Postal 175, C.P. \\ 87010, Cd. Victoria, Tams., MÉXICO. agutierr@uat.edu.mx \\ 2 Department of Biological Sciences, Angelo State University, San Angelo, Texas, 76901, U.S.A.
}

Correa-Sandoval, A., N. E. Strenth, R. Rodríguez Castro y J. V. Horta Vega. 2009. Análisis ecológico básico de los gastrópodos terrestres de la Región Oriental de San Luis Potosí, México. Acta Zool. Mex. (n. s.) 25(1):105-122.

RESUMEN. En este estudio se caracterizaron las condiciones ecológicas en las que se presentaron 77 especies de gastrópodos terrestres en la región oriental de San Luis Potosí, existiendo más especies de moluscos en bosque de encino (44 especies), hojarasca y humus (66), suelos húmedos todo el año (53), precipitaciones de 3000-3500 mm anuales (41) y altitudes de 801-900 m (43). Thysanophora hornii es la de mas amplia distribución en función de los tipos de vegetación, condiciones de humedad, precipitación, tipos de suelo y rango de altitud. Los valores más grandes de diversidad y equitatividad se observaron en el bosque mesófilo de montaña (0.6895) y bosque de encino (0.3512). El mayor valor de dominancia (0.9958) y amplitud ecológica del hábitat (0.3471) se presentó en el matorral xerófilo. Las comunidades de gastrópodos terrestres se encontraron subordinadas ecológicamente a las del bosque tropical caducifolio - subcaducifolio, y a su vez ésta se halla subordinada a la del bosque de encino. El valor de similitud cualitativa más alto se observó entre el bosque tropical caducifolio-subcaducifolio y pastizal-cultivos ( 0.7462$)$. Entre el bosque espinoso y pastizalcultivos se obtuvo el mayor valor de similitud cuantitativa (0.3646).

Palabras clave: Gastropoda terrestres, biodiversidad, San Luis Potosí, región oriental, ecología.

Correa-Sandoval, A., N. E. Strenth, R. Rodríguez Castro \& J. V. Horta Vega. 2009. Basic ecological analysis of the terrestrial gastropods of the eastern region of San Luis Potosí, Mexico. Acta Zool. Mex. (n. s.) 25(1):105-122.

ABSTRACT. Of 77 species of terrestrial gastropods found in the eastern region of the state of San Luis Potosí, the ecological conditions supporting the largest number of species of mollusks are as follows: oak forest (44 species), leaf litter and humus (66), soils which are humid throughout the year (53), 3000$3500 \mathrm{~mm}$ annual rainfall (41) and 801-900 $\mathrm{m}$ altitude (43). Thysanophora hornii exhibits the greatest distribution with regard to vegetational types, humidity conditions, rainfall, soil types, and altitudinal ranges. The greatest values of diversity and evenness were obtained in the cloud forest (0.6895) and oak forest $(0.3512)$ respectively. The chaparral exhibits the greatest dominance (0.9958) and habitat ecological width $(0.3741)$. Mollusks communities were ecologically ranked lower than the tropical decidouos-subdeciduous forest. The highest qualitative similarity was observed between tropical deciduous-subdeciduous forest and savannah-cultivation (0.7462). The greatest quantitative similarity (0.3646) was observed between thorn forest and savannah-cultivation.

Key words: Terrestrial Gastropoda, biodiversity, San Luis Potosí, eastern region, ecology. 


\section{INTRODUCCIÓN}

Los estudios con enfoque exclusivamente ecológico, así como geográfico, son necesarios e importantes al analizar la distribución de los moluscos, particularmente los terrestres (Bequaert \& Miller, 1973, Peake 1978, Cook 2001). Sin embargo, en México son aún escasos los estudios que han revisado especialmente la ecología de los gastrópodos terrestres. La información disponible sobre las preferencias ecológicas de algunos moluscos terrestres se halla dispersa en diferentes trabajos (Naranjo-García 2003a), principalmente taxonómicos, lo cual sucede también para muchas regiones en el mundo.

Entre los estudios ecológicos es importante señalar el realizado en la región de Los Tuxtlas, Veracruz por Álvarez et al. (2003) en donde se analiza la influencia de la acumulación de hojarasca en las poblaciones de moluscos terrestres; y el de Naranjo-García (2003b) que, además de recopilar información de la malacofauna terrestre y su relación con la hojarasca, establece diferencias del número de especies y abundancia de gastrópodos en función de dos tipos de árboles y según la estación seca o lluviosa del año. Particularmente, en el noreste de México, tres estudios sistematizan algunos aspectos ecológicos de los gastrópodos terrestres: CorreaSandoval (1993) en el que se señala que en el área de Santiago, Nuevo León, el tipo de vegetación con más especies (13) es el bosque de Quercus-Pinus-Pseudotsuga; Correa-Sandoval (1996-1997) en el que se indica que el bosque de galería de Populus-Quercus-Juglans, cobertura vegetal total y escasa pendiente, en Iturbide, Nuevo León, son los parámetros ambientales más ricos en especies (11); y el de Correa-Sandoval y Rodríguez-Castro (2005) que establece que el tipo de vegetación en la Reserva de la Biosfera "El Cielo" con mayor número de especies (36) es el bosque tropical subcaducifolio.

Del 50 y $48 \%$ de las especies de gastrópodos conocidas para la región noreste de México y oriental de San Luis Potosí, respectivamente, no se tiene información ecológica (Correa-Sandoval 2003, Correa-Sandoval y Salazar-Rodríguez 2005). Por otra parte, los estudios sinecológicos en caracoles terrestres son escasos (Pérez et al. 1996). El gran valor de los trabajos de Walden (1981) y Cameron (1982) reside en el método propuesto (Pérez et al. 1996).

Para México es necesario mencionar el trabajo realizado en Tabasco por RangelRuiz y Gamboa (2005) en un cuerpo dulceacuícola, en el cual es estudiada la estructura de una comunidad compuesta por especies terrestres (4), dulceacuícolas (4) y una anfibia, y se revisa la dinámica poblacional en relación con la temperatura, precipitación y frecuencia de precipitación.

Los distintos tipos de clima, vegetación y de suelo, así como la variada fisiografía en la región oriental de San Luis Potosí, determinan la presencia de diferentes micro hábitats para la malacofauna terrestre (Correa-Sandoval et al. 1998). Por lo anterior, esta investigación tuvo como propósito caracterizar ecológicamente las comunidades 
de gastrópodos terrestres de esta región, señalando diversos parámetros ambientales en las que se distribuyen las diferentes especies así como algunas de sus características sinecológicas.

La región se ubica entre los paralelos $21^{\circ} 11^{\prime} 55^{\prime}$ " y $23^{\circ} 14^{\prime} 03^{\prime \prime}$ de latitud norte y los meridianos $98^{\circ} 17^{\prime} 53^{\prime \prime}$ y $100^{\circ} 23^{\prime} 39^{\prime \prime}$ de longitud oeste. Es la parte centro occidental de la región Huasteca y posee sierras y valles de importancia forestal y agrícola (Rzedowski 1978, Rivera \& Domínguez 1994, Challenger 1998). Fisiográficamente pertenece a las provincias de la Llanura Costera del Golfo Norte y Sierra Madre Oriental (INEGI 1981a). Los tipos de vegetación que comprende la región de estudio son: matorral xerófilo, bosque espinoso, bosque tropical caducifolio y subcaducifolio, bosque tropical perennifolio, bosque mesófilo de montaña, bosque de encino, y pastizal y cultivos (Rzedowski 1978, Challenger 1998, Contreras-Medina 2004). De acuerdo a la clasificación de Köeppen, el oriente del área de estudio presenta un clima cálido-subhúmedo con lluvias en verano, al centro es semicálido-húmedo y al oeste el clima es semiseco-semicálido con lluvias en verano (INEGI 1981b).

\section{MATERIAL Y MÉTODOS}

En este trabajo se usa la información taxonómica publicada por Correa-Sandoval et al. (1998) para la región de estudio, quienes visitaron 48 localidades entre los meses de junio de 1995 a agosto de 1996, para realizar muestreos (23 por tipo de vegetación) en cuadrantes o parcelas de $1 \mathrm{~m}^{2}$ obtenidos al azar, siguiendo las recomendaciones específicas de Santos y Hairston (1956), Coney et al. (1981) y Lavelle et al. (1981) para el estudio de moluscos terrestres y macrofauna del suelo. El número de cuadrantes por tipo de vegetación y las dimensiones de los mismos son óptimos como áreas mínimas de muestreo en gastrópodos terrestres ya que permiten revisar exhaustivamente el área trabajada (Pérez et al. 1996, Correa-Sandoval 1997). En cada cuadrante se recogieron en forma directa ejemplares macroscópicos y se inspeccionaron rocas y las cortezas de árboles hasta dos metros de altura. El suelo suelto, que varió en su peso de dos a cuatro kilogramos aproximadamente en cada una de las parcelas, se tamizó y revisó en el laboratorio de acuerdo a Cameron y Redfern (1976). El trabajo de campo fue realizado por cinco personas y el tiempo aproximado de muestreo en cada cuadrante fue de una hora. Los animales se narcotizaron en solución acuosa mentolada y se conservaron en alcohol etílico al 70 $\%$ (Solem et al. 1980). Se revisaron en total 1659 lotes (11,546 ejemplares), los cuales están depositados en las colecciones del Instituto Tecnológico de Ciudad Victoria, Tams. y Florida Museum of Natural History, University of Florida. Duplicados de algunas especies se encuentran en el Instituto de Biología e Instituto de Ciencias del Mar y Limnología de la Universidad Nacional Autónoma de México.

En los resultados, el arreglo de las familias sigue la propuesta de Hubricht (1985), de Taylor y Sohl (1962) y del Dr. Fred G. Thompson en la Colección Malacológica 
del Florida Museum of Natural History. Los géneros y especies están ordenados alfabéticamente.

Se observó qué especies de gastrópodos terrestres se encontraron según cada tipo de vegetación (hábitat primario a considerar), tipo de suelo, meses húmedos, precipitación y altitud de acuerdo a los criterios para establecer estos parámetros de Rzedowski (1978), Dillon (1980), INEGI (1980a, 1980b, 1981c, 1981d) y de acuerdo también a las observaciones de campo.

Para las comunidades de moluscos por tipo de vegetación se estimó la diversidad de Shannon-Wienner según Krebs (1989), se obtuvo el número de especies (McIntosh 1967, Krebs 1989) y se calculó la Equitatividad (Pielou 1975, 1977); se estimaron además la amplitud ecológica del hábitat y subordinación ecológica (con valores de referencia de 66.66 \%) según Pérez et al. (1996) y dominancia según McNaughton y Wolf (1970).

Se obtuvo también el índice biogeográfico de las comunidades de gastrópodos en función de las afinidades zoogeográficas de cada especie de acuerdo a los criterios de Bequaert y Miller (1973), Correa-Sandoval (1997, 1999) y Correa-Sandoval et al. (2007).

Para analizar la similitud cualitativa de las malacofaunas terrestres entre los hábitats se empleó el índice de Sorensen (1948). La similitud cuantitativa de las comunidades de gastrópodos terrestres entre los hábitats se estudió mediante el índice métrico de Canberra (Lance \& Williams 1966) considerando los valores de abundancia relativa de cada especie dentro de la comunidad, de acuerdo a las recomendaciones de Hutta (1979). Se elaboraron dendrogramas de similitud siguiendo el método de ligamiento promedio de acuerdo a Cody (1974), Crisci y López-Armengol (1983) y Krebs (1989).

\section{RESULTADOS}

Distribución de los gastrópodos terrestres por condiciones ecológicas. De 77 especies de moluscos terrestres halladas en la región, los tipos de vegetación con mayor número de especies de caracoles fueron el bosque de encino (44), bosque tropical caducifolio-subcaducifolio (42) y mesófilo de montaña (41) (Cuadro 1, Apéndice 1). El matorral xerófilo fue el que presentó menos especies (12).

En hojarasca y humus se encontraron 66 especies de gastrópodos y en suelos arcillosos o lodosos 40. Los suelos húmedos todo el año presentaron el mayor número de especies (53) y los que tienen ocho a diez meses de humedad presentaron 40 . Zonas con precipitación de 3000 - $3500 \mathrm{~mm}$ al año presentaron el mayor número de especies (41). El intervalo de altitud con más especies de gastrópodos terrestres fue el de los 801 - 900 m con 43.

Particularmente, Thysanophora hornii fue la especie más ampliamente distribuida en la región de estudio en función de las características del hábitat: en todos los tipos de vegetación, condiciones diferentes de humedad y precipitación, en cuatro diferentes tipos de suelo y en once rangos de altitud (Apéndice 1). 
Cuadro 1. Índices ecológicos obtenidos en las comunidades de moluscos terrestres por tipo de vegetación: Diversidad (H'), Riqueza de especies (S), Equitatividad (J'), Amplitud ecológica del hábitat $(\mathrm{AEH})$, Dominancia de Mc Naughton y Wolf $\left(\mathrm{D}_{1,2}\right)$, Índice Biogeográfico (IB, en paréntesis el número de especies endémicas). Simbología de vegetación del Apéndice 1.

\begin{tabular}{lccccccc}
\hline $\begin{array}{l}\text { Tipo de } \\
\text { vegetación }\end{array}$ & $\mathrm{H}^{\prime}$ & $\mathrm{S}$ & $\mathrm{J}$ & $\mathrm{AEH}$ & $\mathrm{D}_{1,2}$ & $\mathrm{IB}$ & \\
\hline MX & 0.0489 & 12 & 0.0613 & 0.3471 & 0.9958 & 4.25 & $(3)$ \\
BE & 0.3285 & 15 & 0.2304 & 0.3333 & 0.8155 & 3.6 & $(0)$ \\
BTCS & 0.5096 & 42 & 0.2895 & 0.0906 & 0.7495 & 4.55 & $(10)$ \\
BTP & 0.6376 & 29 & 0.3510 & 0.1449 & 0.6472 & 4.31 & $(1)$ \\
BMM & 0.6895 & 41 & 0.3176 & 0.0825 & 0.6024 & 4.75 & $(13)$ \\
BEN & 0.6589 & 44 & 0.3512 & 0.0825 & 0.6709 & 4.70 & $(17)$ \\
PC & 0.2013 & 25 & 0.1214 & 0.1920 & 0.8228 & 4.40 & $(4)$ \\
\hline
\end{tabular}

Pupisoma dioscoricola insigne también se presentó en todos los tipos de vegetación y en cuatro tipos de suelo, pero en menos condiciones (siete) de humedad, precipitación e intervalos de altitud. Cecilioides consobrina veracruzensis es la segunda especie mejor distribuida en función de la altitud (10 rangos de altitud).

Entre las especies que tuvieron una distribución restringida en relación con los parámetros ecológicos (en uno sólo de cada tipo) en la región de estudio se encontraron Schasicheila xanthia, Aperostoma mexicanum palmeri, Adelopoma stolli y Leidyula moreleti.

Organización estructural. Los valores de los índices ecológicos calculados para las comunidades malacofaunísticas se presentan en el Cuadro 1. Los mayores valores de diversidad se observaron en el bosque mesófilo de montaña (0.6895) y en el bosque de encino (0.6589). Los valores más altos de equitatividad se presentaron en el bosque de encino (0.3512) y en el bosque tropical perennifolio (0.3510). Los valores de amplitud ecológica del hábitat resultaron mayores para el matorral xerófilo (0.3471) y el bosque espinoso (0.3333). El mayor valor de dominancia se presentó en el matorral xerófilo (0.9958).

La mayor parte de los valores de subordinación ecológica fueron mayores al de referencia (66.66) (Fig. 1). Las comunidades de moluscos de cuatro tipos de vegetación (bosque espinoso, tropical perennifolio, matorral xerófilo y pastizalcultivos) se encontraron subordinadas al bosque tropical caducifolio-subcaducifolio. La subordinación fue total en el caso del pastizal-cultivos.

Los mayores valores del índice biogeográfico se obtuvieron en el bosque mesófilo de montaña (4.75) y en bosque de encino (4.70). 


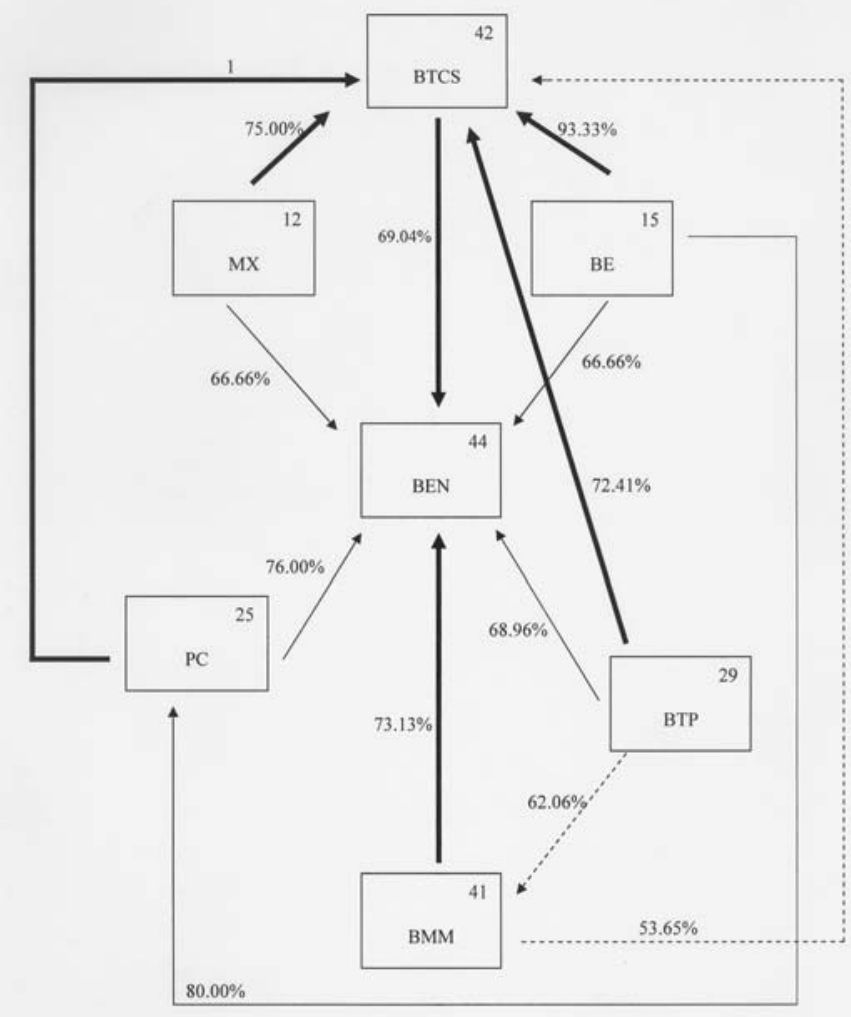

Figura 1. Relaciones de subordinación ecológica entre las comunidades de gastrópodos terrestres por tipos de vegetación en la región oriental de San Luis Potosí. Las saetas en las flechas indican el sentido de la subordinación. Las flechas engrosadas marcan la principal subordinación. Con líneas punteadas las subordinaciones menores a $66.66 \%$. En la esquina superior derecha de los cuadros el número de especies en cada tipo de vegetación. La simbología es del Apéndice 1.

Análisis de clasificación entre las comunidades de moluscos. De acuerdo con los valores de similitud cualitativa se establecieron dos grupos (Fig. 2) con los valores más altos: bosque tropical caducifolio-subcaducifolio y pastizal-cultivos (0.7462) y entre bosque mesófilo de montaña y bosque de encino (0.7058). Las mayores similitudes cuantitativas (Fig. 3) se obtuvieron entre el bosque espinoso y pastizal-cultivos (0.3646) y entre el bosque tropical caducifolio-subcaducifolio y el bosque de encino (0.2850). 


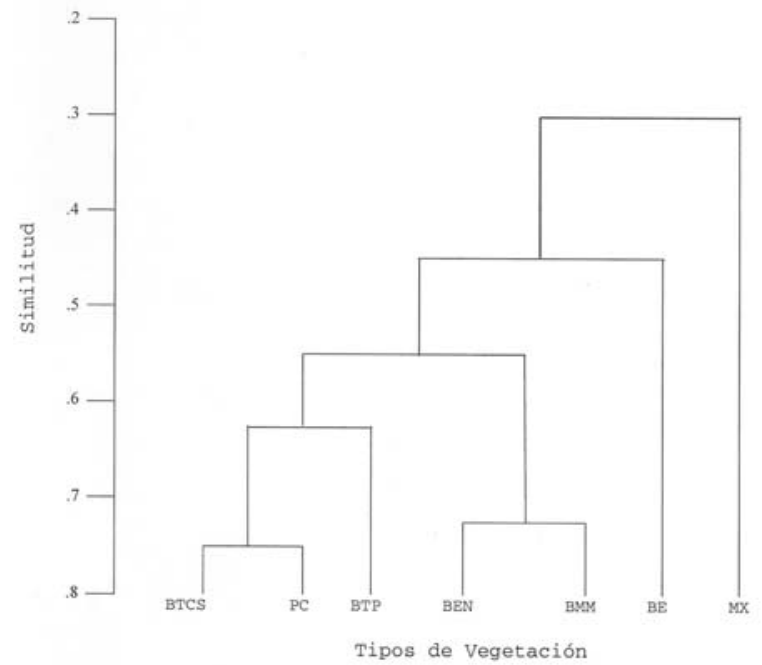

Figura 2. Similitud cualitativa entre las comunidades de moluscos terrestres por tipos de vegetación en la región oriental de San Luis Potosí. La simbología es del Apéndice 1.

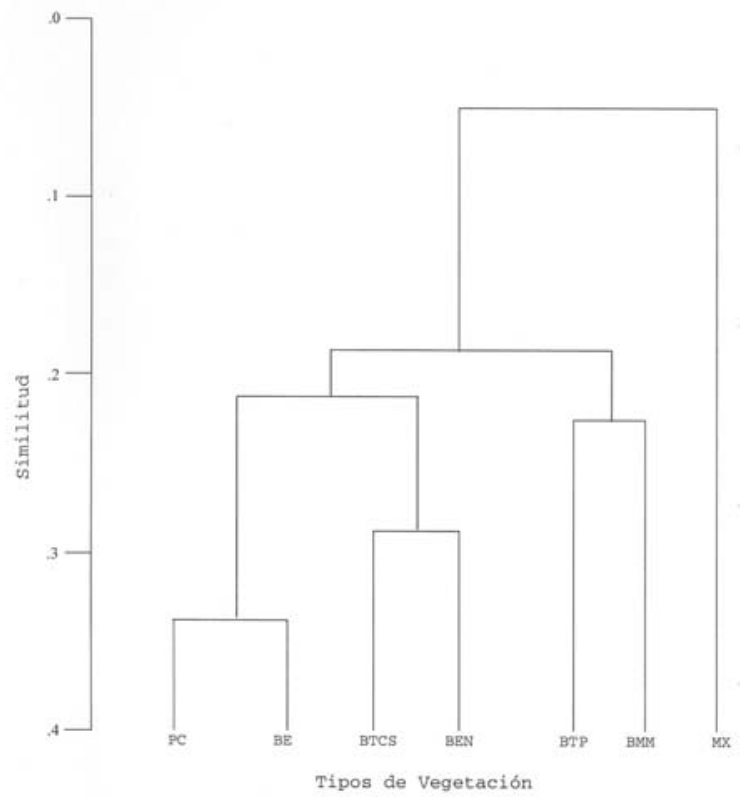

Figura 3. Similitud cuantitativa entre las comunidades de gastrópodos terrestres por tipos de vegetación en la región oriental de San Luis Potosí. La simbología es del Apéndice 1. 


\section{DISCUSIÓN}

Distribución por condiciones ecológicas. Considerando que diferentes características del hábitat determinan la distribución (Cook 2001) y diversidad de los moluscos terrestres, la importancia de algunos parámetros como el tipo de vegetación (Karlin 1961, Hunter 1964, Tattesfield 1996, Correa-Sandoval 1996-1997, Tattlesfield et al. 2001), es evidente en el oriente de San Luis Potosí. El mayor número de especies de gastrópodos terrestres se encontraron en el bosque de encino (57.4\% del total de especies de caracoles), como en el bosque tropical caducifoliosubcaducifolio (54.5\%) y en el bosque mesófilo de montaña (53.2\%), los cuales proporcionan considerable cobertura sobre el suelo, protección contra la desecación, abundante hojarasca y alimento, lo que favorece la presencia de estos organismos (Boycott 1934, Berazain 1981, Álvarez-Sánchez et al. 2003).

Por el contrario, el menor número de especies de moluscos terrestres hallados en el matorral xerófilo, se explica por la escasa cobertura vegetal, bajas precipitaciones y humedad, y más iluminación sobre el suelo (Hunter 1964, INEGI 1980b, 1981d, Correa-Sandoval et al. 2007).

Tomando en cuenta que el tipo de suelo puede afectar la distribución o la abundancia de los moluscos terrestres (Bequaert \& Miller 1973, Wilhelm 1976, Correa-Sandoval 1993, Álvarez-Sánchez et al. 2003), lo importante es establecer en qué grado se presenta esta influencia en diferentes áreas de estudio.

La mayor parte de las especies de moluscos terrestres halladas en Los Tuxtlas, Veracruz, se presentan en la hojarasca (Álvarez-Sánchez et al. 2003). Esto coincide en parte en la región de estudio, en donde un elevado número de especies (el 91.6\% del total) se presentan en hojarasca y humus. Esto se debe a que la hojarasca proporciona un clima adecuado, refugios y alimento a diversas especies, por lo que incide en su distribución (Petersen \& Luxton 1982, Solem \& Climo 1985, Locasciulli \& Boag 1987, Thompson \& Correa-Sandoval 1994).

Los suelos húmedos todo el año presentan el $68.8 \%$ de especies lo cual se explica en función de que las condiciones de alta humedad favorecen la actividad, distribución y la diversidad de los gastrópodos terrestres (Berry 1963, Hunter 1964, Hyman 1967, Boag 1985, Monge-Nájera 1997). Lo anterior coincide con lo hallado para otras zonas cercanas en la región como lo es el sur de Nuevo León (CorreaSandoval 1993, 1996-1997, Martínez-Ramírez 2004).

En parte, la escasa precipitación pluvial al noroccidente de la región de estudio (INEGI 1980b) explica el menor número de especies de caracoles terrestres (12). En esta zona se presenta matorral xerófilo, el cual proporciona una cobertura vegetal reducida que afecta negativamente la presencia de los moluscos (Dillon 1980). Por el contrario, por el efecto combinado de los factores ambientales (mayor precipitación, tipos de vegetación con mas follaje, mayor cobertura vegetal) el bosque de encino y el bosque tropical caducifolio-subcaducifolio son los más ricos 
en especies. En estos ambientes se presentan precipitaciones promedio mensuales de 116 a $166 \mathrm{~mm}$ (INEGI 1980b). Una buena referencia comparativa se conoce de Nueva Zelanda, en donde precipitaciones promedio mensuales de 90 a $160 \mathrm{~mm}$ es uno de los parámetros más importantes para explicar la presencia de los moluscos terrestres (Solem 1984). Sin embargo, en la zona sureste de la región de estudio, en donde se hallan precipitaciones promedio mensuales superiores (de 250 a $290 \mathrm{~mm}$ ) (INEGI 1980b), en el bosque mesófilo de montaña, también se encuentra un elevado número de especies (41) de gastrópodos terrestres. Esto también puede explicarse por tratarse de un hábitat sombreado, con abundante hojarasca, suelo húmico y refugios (Fretter 1975, Boag 1985, Solem 1984, Luis de Vasconcelos 1990).

El $59 \%$ de la malacofauna terrestre del oriente de San Luis Potosí se presenta entre los 800 y 900 m de altitud. Sin embargo, en un intervalo mayor (1500 a 2000 $\mathrm{m})$, en una zona cercana como el sur de Nuevo León, en donde se presentan varios tipos de vegetación similares, se halló el mayor número de especies $(42.84 \%)$ de acuerdo a Martínez-Ramírez (2004). Esto significa que la altitud contribuye a explicar sólo en parte la presencia y la distribución geográfica de los gastrópodos terrestres, y que debe ser considerada conjuntamente con diversos parámetros ambientales como son el tipo de vegetación y de suelo, entre otros.

Los gastrópodos terrestres se presentan en un amplio rango de condiciones ecológicas (Heller 2001). Esto es evidente a nivel de especies en Thysanophora hornii, que parece no tener preferencias por un determinado hábitat (Naranjo-García 2003b, Pérez \& López 1993). Esta especie está adaptada a una amplia variedad de ambientes, tanto en los muy áridos, como en los mesofíticos moderadamente húmedos (Bequaert \& Miller 1973, Hubricht 1985) al igual que en los bosques tropicales caducifolios y subcaducifolios (Naranjo-García 2003b). Es indiferente a la naturaleza del terreno, suelo o tipo de vegetación, y frecuentemente se le encuentra donde otros moluscos terrestres no pueden sobrevivir (Bequaert \& Miller 1973).

Lo anterior se confirma claramente al presentarse en el mayor número de ambientes presentes en la región oriental de San Luis Potosí y ser una de las especies, junto con Praticolella berlandieriana, de más amplia distribución geográfica en el noreste de México (Correa-Sandoval 1996-1997, 2000, 2003, y Correa-Sandoval \& Salazar-Rodríguez 2005).

Pupisoma dioscoricola insigne, presente en la mayor parte de las diferentes condiciones ecológicas en la región de estudio, es una de las especies, al igual que Gastrocopta pellucida, con más amplia distribución por tipos de vegetación, suelo, condiciones de humedad y altitud en el sur de Nuevo León (Martínez-Ramírez 2004). La clara condición generalista en términos ecológicos (Brown \& Gibson 1983) también es notable en Cecilioides consobrina veracruzensis, la cual posee una amplia distribución en los diferentes bosques tropicales del noreste de México (Correa-Sandoval 1999, Correa-Sandoval et al. 2007). Una condición ecológica 
contraria, la especialista, explica la distribución restringida en la zona de Schasicheila xanthia, Aperostoma mexicanum palmeri, Adelopoma stolli, Leidyula moreleti, Carychium mexicanum y Streptostyla potosiana, las cuales requieren suelos muy húmedos, abundante hojarasca y humus. En el caso de Euglandina lamyi, se halla adaptada exclusivamente al matorral xerófilo, y a la escasa humedad y precipitación.

Organización estructural y análisis de clasificación. Los valores que se obtienen a partir del uso de los índices ecológicos no siempre son fáciles de interpretar (Ludwig \& Reynolds 1988). Sin embargo permiten tener una idea de la estructura de las comunidades estudiadas (Pérez et al. 1996). En el área de estudio los mayores valores de diversidad obtenidos en bosque mesófilo de montaña y bosque de encino se explican porque estos tipos de vegetación, que son de los que poseen más especies de gastrópodos terrestres, presentan, además de la diversidad de microhábitats para los moluscos, múltiples factores ecológicos que los benefician como cobertura vegetal, disponibilidad de alimento, humedad y precipitación, que son relevantes para las comunidades de estos organismos (Burch 1955, Basch et al. 1961, Solem \& Climo 1985).

Los mayores valores de equitatividad que se presentan en el bosque de encino, bosque tropical perennifolio y bosque mesófilo de montaña, pueden explicarse precisamente por la baja dominancia de los moluscos terrestres. Por el contrario, en el matorral xerófilo, en pastizal-cultivos y en bosque espinoso, se presentaron los mayores valores de dominancia de caracoles y los menores valores de diversidad y equitatividad, ya que frecuentemente sólo dos especies, Gastrocopta pellucida y Praticolella berlandieriana, aparecieron como únicas especies en los muestreos.

Algo similar ocurre en algunas formaciones vegetales de Cuba como el Cuabal y la manigua costera (Berazain 1981, Capote \& Berazain 1984) donde los menores valores de equitatividad y diversidad se explican por la dominancia de Subulina octona Brugiére y Gongylostoma elegans (Pilsbry), al presentarse factores limitantes para las comunidades de gastrópodos terrestres como la escasa cobertura vegetal, baja humedad y temperaturas más elevadas.

En el bosque tropical caducifolio-subcaducifolio, bosque de encino y bosque mesófilo de montaña, en donde se presentó la mayor riqueza de especies y valores bajos de amplitud ecológica del hábitat, estos se explican por la presencia del núcleo de especies con mayor especialización en función del hábitat.

Los mayores valores de precipitación y la humedad asociada, pero especialmente la abundancia de humus y hojarasca, explican en parte la subordinación de las comunidades de gastrópodos de varios tipos de vegetación a la del bosque tropical caducifolio-subcaducifolio, y a su vez de este y del bosque mesófilo de montaña a la del bosque de encino. Los valores más altos de subordinación al bosque tropical caducifolio-subcaducifolio, así como el establecimiento de uno de los dos grupos con 
base en el índice de similitud cualitativa (Fig. 2), probablemente se explica por la proximidad entre sí del bosque tropical mencionado y el pastizal-cultivos. Esto sucede también entre el bosque seco-bosque semideciduo y la manigua costera en Cuba, donde las comunidades de gastrópodos terrestres se encuentran próximas entre sí, siendo factible el desplazamiento de los animales entre éstas (Pérez et al. 1996). Debe señalarse además que, en la región de estudio, el total de las especies (25) presentes en pastizal-cultivos se hallan en el bosque tropical caducifoliosubcaducifolio. Treinta especies (el valor más alto de especies en común entre las diferentes comunidades de moluscos) se presentaron entre el bosque mesófilo de montaña y el bosque de encino, lo cual explica el segundo grupo formado.

El que las comunidades malacológicas del matorral xerófilo y bosque espinoso se hallen apartadas de las demás, y se muestren como unidades dependientes de las comunidades del bosque tropical caducifolio-subcaducifolio y del bosque de encino (Fig. 1 y 2) es expresión en parte, de la alta capacidad adaptativa de las especies que las integran. La disimilitud de estas comunidades de gastrópodos con las demás se debe a una menor riqueza de especies y no a la ausencia de especies comunes (Pérez et al. 1996, Correa-Sandoval 1997).

Al considerar las abundancias relativas (Fig. 3), la mayor relación entre el bosque espinoso y pastizal-cultivos se explica por la abundancia de Polygyra oppilata y Cecilioides consobrina veracruzensis en ambos tipos de vegetación, aunque, como ya se mencionó, son otras dos especies (Gastrocopta pellucida y Praticolella berlandieriana) las más abundantes en estos tipos de vegetación. La segunda mayor relación entre el bosque tropical caducifolio-subcaducifolio y el bosque de encino se debe a la abundancia en ambos de Guppya gundlachi.

En el bosque mesófilo de montaña y en el bosque de encino se hallan los dos principales núcleos de especies endémicas (13 y 17 especies respectivamente) (Correa-Sandoval 1999, Correa-Sandoval et al. 1998), lo cual explica su mayor valor biogeográfico. Esto coincide con lo encontrado para la vegetación de galería en el sur de Nuevo León en donde se presentaron más especies endémicas (Correa-Sandoval et al. 2007). Los anteriores tipos de vegetación propician la presencia de la malacofauna terrestre al proporcionar hábitats sombreados, suelos húmicos, abundante hojarasca, refugios y elevada humedad. Como lo indican Pérez et al. (1996) y Correa-Sandoval et al. (2007), las áreas con mayor valor biogeográfico en función de sus endemismos deben ser prioritarias para la conservación.

AGRADECIMIENTOS. A Fred G. Thompson (Florida Museum of Natural History, University of Florida), Art L. Metcalf (University of Texas, El Paso) y Raymond W. Neck (Texas Parks and Wild Life Department) por la literatura proporcionada y comentarios al estudio. Al Consejo Nacional de Ciencia y Tecnología por el apoyo económico otorgado. A Anabel Gutiérrez por su asistencia en la realización de este trabajo. A Antonio García-Cubas Gutiérrez, María Martha Reguero Reza y Blanca Estela Buitrón Sánchez por su constante interés y observaciones al estudio. A Edna Naranjo-García por el envío de sus 
publicaciones. A Víctor Martínez, Lauro Valladares, Enrique Gudiño y Edgar Camacho quienes participaron en el trabajo de campo y laboratorio. A dos revisores anónimos por las correcciones, sugerencias y comentarios que mejoraron sustancialmente el manuscrito.

\section{LITERATURA CITADA}

Álvarez-Sánchez, J., E. Naranjo-García \& S. Guevara. 2003. Perspectivas de estudios de acumulación de hojarasca en el subdosel y animales asociados. El caso de los moluscos. Pp. 80-87. In. J. Álvarez-Sánchez \& E. Naranjo-García (Eds.). Ecología del suelo en la selva tropical húmeda de México. Instituto de Ecología, A. C.- Universidad Nacional Autónoma de México. Xalapa, México.

Basch, P. F., P. Bainer \& J. Wilhm. 1961. Some ecological characteristics of the molluscan fauna of a typical grassland situation in east central Kansas. American Midland Naturalist 66(1): 178 -199.

Bequaert, J. C. \& W. B. Miller. 1973. The mollusks of the arid southwest with an Arizona check list. University of Arizona Press. U.S.A. 271 pp.

Berazain, R. 1981. La zona de vegetación serpentinícola del Jardín Botánico Nacional. Revista del Jardín Botánico Nacional 2: 151-181.

Berry, A. J. 1963. Growth and variation of the shell in certain malayan limestone hill snails. Proceedings of the Malacological Society 35:203-206.

Boag, D. A. 1985. Microdistribution of three genera of small terrestrial snails (Stylommatophora: Pulmonata). Canadian Journal of Zoology 63(5): 1089-1095.

Boycott, A. C. 1934. The habitat of land mollusca in Brittain. Journal of Ecology 22: 1-38.

Brown, J. H. \& A. C. Gibson. 1983. Biogeography. C. V. Mosby Co. St. Louis Missouri. 643 pp.

Burch, J. B. 1955. Some ecological factors of the soil affecting the distribution and abundance of the land snails in eastern Virgina. Nautilus 69(2): 62 - 69.

Cameron, R. A. D. 1982. Life histories, density and biomass in a woodland snail community. Journal of Molluscan Studies 48: 159-166.

Cameron, R. A. D. \& M. Redfern. 1976. A synopsis of the British land snails. Academic, Londres: 9.

Capote, R. \& R. Berazain. 1984. Clasificación de las formaciones vegetales de Cuba. Revista del Jardín Botánico Nacional 5: 27-76.

Cody, M. L. 1974. Competition and the structure of bird communities. Princeton University Press. New Jersey. 318 pp.

Conney, C. L., W. A. Tarpley \& R. Bohannan. 1981. A method of collecting minute land snails. Nautilus 95(1): 43-44.

Contreras-Medina, R. 2004. Gimnospermas. Pp. 137-148. In: I. Luna, J. J. Morrone \& D. Espinosa (Eds.). Biodiversidad de la Sierra Madre Oriental. Comisión Nacional para el Conocimiento y Uso de la Biodiversidad - Universidad Nacional Autónoma de México. México, D. F. 527 pp.

Cook, A. 2001. Behavioural ecology: on doing the right thing, in the right place at the right time. Pp. 447-487. In: G. M. Barker (Ed.). The biology of terrestrial molluscs. CABI Publishing. Oxon.

Correa-Sandoval, A. 1993. Caracoles terrestres (Mollusca: Gastropoda) de Santiago, Nuevo León, México. Revista de Biología Tropical 41: 683-687.

—. 1996-1997. Caracoles terrestres (Mollusca:Gastropoda) de Iturbide, Nuevo León, México. Revista de Biología Tropical 44(3)/45(1): 137-142.

. 1997. Composición taxonómica, estructura y zoogeografía de las comunidades de gastrópodos terrestres de la región oriental de San Luis Potosí, México. Tesis Doctoral. Facultad de Ciencias. Universidad Nacional Autónoma de México. México, D. F.157 pp.

. 1999. Zoogeografía de los gastrópodos terrestres de la región oriental de San Luis Potosí, México. Revista de Biología Tropical 47(3): 493-502. 

s.) 79: 1-9.

2000. Gastrópodos terrestres del norte de Veracruz, México. Acta Zoológica Mexicana (n. 2003. Gastrópodos terrestres del noreste de México. Revista de Biología Tropical 51 (Suppl. 3.): 507-522.

Correa-Sandoval, A., A. García-Cubas \& M. Reguero. 1998. Gastrópodos terrestres de la región oriental de San Luis Potosí, México. Acta Zoológica Mexicana (n. s.) 73: 1-17.

Correa-Sandoval, A. \& M. C. Salazar-Rodríguez. 2005. Gastrópodos terrestres del sur de Nuevo León, México. Acta Zoológica Mexicana (n. s.) 21(2): 51-61.

Correa-Sandoval, A., N. E. Strenth \& M. C. Salazar-Rodríguez. 2007. Zoogeografía de los gastrópodos terrestres del Sur de Nuevo León, México. Acta Zoológica Mexicana (n. s.). 23(2): 143-162.

Correa-Sandoval, A. \& R. Rodríguez-Castro. 2005. Los gastrópodos terrestres. Pp. 340-344. In. G. Sánchez-Ramos, P. Reyes-Castillo \& R. Dirzo (Eds.). Historia Natural de la Reserva de la Biosfera El Cielo, Tamaulipas, México. Universidad Autónoma de Tamaulipas. Hong Kong. 732 pp.

Crisci, J. V. \& M. F. López-Armengol. 1983. Introducción a la teoría y práctica de la taxonomía numérica. OEA, Serie Biología, núm. 26. Washington, D. C. 132 pp.

Challenger, A. 1998. Utilización y conservación de los ecosistemas terrestres de México. Pasado, presente y futuro. Comisión Nacional para el Conocimiento y Uso de la Biodiversidad- Universidad Nacional Autónoma de México - Agrupación Sierra Madre, S. C. México, D. F. 847 pp.

Dillon, R. T. 1980. Multivariate analysis of desert snail distribution in an Arizona canyon. Malacologia 19(2): 201-207.

Fretter, V. 1975. Introduction. 1: XI-XXIX In: V. Fretter \& J. Peake (Eds.). Pulmonates. Functional anatomy and physiology. Academic Press. London.

Heller, J. 2001. Life history strategies. Pp. 413-455. In: G. M. Barker (Ed.). The biology of terrestrial molluscs. CABI Publishing. Oxon.

Hubricht, L. 1985. The distribution of the native land mollusks of the eastern United States. Fieldiana: Zoology, New Series 24: 1-191 pp.

Hunter, W. R. 1964. Physiological aspect of ecology in non marine molluscs. Pp. 83-126. In: K. M. Wilbour \& C. M. Yonge (Eds.). Physiology of Mollusca. Vol. 1. Academic Press. New York.

Hutta, V. 1979. Evaluation of different similarity indices as measures of succession in arthropod communities of the forest floor after clear-cutting. Oecología 4: 11-13.

Hyman, L. H. 1967. The Invertebrates: Mollusca I. Vol. 6. Mc Graw - Hill. New York. 792 pp.

Instituto Nacional de Estadística, Geografía e Informática (INEGI). 1980a. Carta de uso de suelo y vegetación. Esc. 1: 1000,000. Secretaría de Programación y Presupuesto. México.

1980b. Carta de precipitación total anual. Esc. 1: 1000,000. Secretaría de Programación y Presupuesto. México.

- 1981a. Carta estatal de regionalización fisiográfica. Esc. 1: 1000,000. Secretaría de Programación y Presupuesto. México.

. 1981b. Carta estatal de climas. Esc. 1:1000,000. Secretaría de Programación y Presupuesto. México.

. 1981c. Carta edafológica. Esc. 1:000,000. Secretaría de Programación y Presupuesto. México. puesto. México.

Karlin, E. J. 1961. Ecological relationships between vegetation and distribution of land snails in Montana, Colorado and New Mexico. American Midland Naturalist 65(1): 60-66.

Krebs, C. 1989. Ecological methodology. Harper Collins Publishers. New York. 654 pp. 
Lance, G. N. \& W. T. Williams. 1966. Computer programs for hierachical polythetic classification ("similarity analysis"). Computer Journal 9: 60 - 64 .

Lavelle, P., M. E. Maury \& V. Serrano. 1981. Estudio cuantitativo de la fauna del suelo en la región de Laguna Verde, Veracruz. Época de lluvias. In. P. Reyes-Castillo (Ed.). Estudios ecológicos en el trópico mexicano. Instituto de Ecología, A. C. No. 6:73-105.

Locasciulli, O. \& D. A. Boag. 1987. Microdistribution of terrestrial snails (Stylommatophora) in forest litter. Canadian Field-Naturalist 10(1): 76-81.

Ludwig, J. A. \& J. F. Reynolds. 1988. Statistical ecology. A primer on methods and computing. Wiley. New York. 337 pp.

Luis De Vasconcelos, H. 1990. Effects of litter collection by understory palms on the association macroinvertebrate fauna in Central Amazonia. Pedobiología 34: 157-160.

Martínez-Ramírez, V. H. 2004. Sinecología de los gastrópodos terrestres del Sur de Nuevo León, México. Tesis Maestría en Ciencias. Instituto Tecnológico de Ciudad Victoria. Ciudad Victoria, México. 84 pp.

McIntosh, R. P. 1967. An index of diversity and the relation of certain concepts to diversity. Ecology 48: 392-404.

McNaughton, S. J. \& L. C. Wolf. 1970. Dominance and the niche in ecological systems. Science 167: 131-139.

Monge-Nájera, J. 1997. Moluscos: moluscos de importancia agrícola y sanitaria en el trópico: la experiencia costarricense. Editorial de la Universidad de Costa Rica. San José, C. R. 166 pp.

Naranjo-García, E. 2003a. Moluscos continentales de México: Terrestres. Revista de Biología Tropical 51 (Suppl. 3): 483-493.

2003b. Malacofauna de la hojarasca. Pp. 141-161. In: J. Alvarez-Sánchez \& E. NaranjoGarcía (Eds.). Ecología del suelo en la selva tropical húmeda de México. Instituto de Ecología, A .C. - Universidad Nacional Autónoma de México. Xalapa, México.

Peake, J. 1978. Distribution and ecology of the Stylommatophora. Pp. 430-495. In: V. Fretter \& J. Peake (Eds.). Pulmonates. 2A. Systematics, evolution and ecology. Academic Press. London.

Pérez, A. M. \& A. López. 1993. Nuevos registros para la fauna malacológica terrestre y fluviátil de Nicaragua. Revista de Biología Tropical 41 (3): 913-915.

Pérez, A. M., J. C. Villaseca \& N. Zione. 1996. Sinecología básica de moluscos terrestres en cuatro formaciones vegetales de Cuba. Revista de Biología Tropical 44(1): 133-146.

Petersen, H. \& M. Luxton. 1982. A comparative analysis of soil fauna populations and their role in decomposition processes. Oikos 39(3): 287-388.

Pielou, E. C. 1975. Ecological diversity. Wiley, New York. 162 pp.

Pielou, E. C. 1977. Mathematical ecology. Wiley, New York. 385 pp.

Rangel-Ruiz, L. J. \& J. Gamboa A. 2005. Estructura de la comunidad y dinámica poblacional de gasterópodos en una zona enzoótica de fasciolosis en Tabasco, México. Acta Zoológica Mexicana (n. s.) 21(2): 79-85.

Rivera, F. \& F. Domínguez. 1994. La Huasteca Potosina: bastión de riqueza biótica. Información Científica y Tecnológica 16 (210): 32-35.

Rzedowski, J. 1978. Vegetación de México. Primera Edición. Editorial Limusa. México. 432 pp.

Santos, B. \& N. G. Hairston. 1956. Quartely and annual field reports of the Philippine schistosomiasis project. Palo Leyte. (Mimeographed document).

Solem, A. 1984. A world model of land snail diversity and abundance. Pp 6-22. In. A. Solem \& A. C. Bruggen (Eds.). World wide snails. Biogeographical studies on non-marine mollusca. Brill, Leiden. $261 \mathrm{pp}$.

Solem, A. \& F. M. Climo. 1985. Structure and habitat correlations of sympatric New Zealand land snails species. Malacologia 26(1-2): 1-30. 
Solem, A., W. K. Emerson, B. Roth \& F. G. Thompson. 1980. Standards for malacological collections. Curator 24(1): 19-28.

Sorensen, T. 1948. A method of stablishing groups of equal amplitude in plant sociology, based on similarity of species content. Kong. Danske Vidensk. Selsk. Biol. Skr. 5 (4): 1-34.

Tattersfield, P. 1996. Local patterns of land snail diversity in a Kenyan rainforest. Malacología 38(12): 161-180.

Tattersfield, P., C. M. Waruj, M. B. Seddon \& J. W. Kiringe. 2001. Land-snails faunas of afromontane forest of Mount Kenya, Kenya: ecology, diversity and distribution patterns. Journal of Biogeography 28(7): 843-861.

Taylor, D. W. \& N. F. Sohl. 1962. An outline of gastropod classification. Malacologia 1(1): 7-32.

Thompson, F. G. \& A. Correa-Sandoval. 1994. Land snails of the genus Coelocentrum from northeastern México. Bulletin of Florida Museum of Natural History, Biological Sciences 36(5): 141-173.

Walden, H. 1981. Communities and diversity of land molluscs in scandinavian woodlands. I. High diversity communities in taluses and boulder slopes in S. W. Sweden. Journal of Conchology 30: 351-377.

Wilhelm, K. 1976. Soil biology, with special reference to the animal kingdom. Faber \& Faber Limited. London. $483 \mathrm{pp}$.

\section{APÉNDICE 1}

Distribución de los gastrópodos terrestres de la región oriental de San Luis Potosí según el tipo de vegetación (matorral xerófilo: MX; bosque espinoso: BE; bosque tropical caducifolio-subcaducifolio: BTCS; bosque tropical perenifolio: BTP; bosque mesófilo de montaña: BMM; bosque de encino: BEN; pastizal-cultivos: PC), tipo de suelo (roca: TS1; suelo pedregoso, con diámetro mayor de $25 \mathrm{cms}$ : TS2; suelo de pedregosidad fina, diámetro menor de 25 cms: TS3; arena: TS4; arcilla o lodo: TS5; humus y hojarasca: TS6), humedad del suelo, en meses (ningún mes: HA; 2-3 meses: HB; 1-6 meses: HC; 5-6 meses: HD; 7-10 meses: HE; 6-11 meses: HF; 8-9 meses: HG; 8-10 meses: $\mathrm{HH}$; húmedo todo el año: HI), precipitación en mm (300-400: P1; 500-600: P2; 600-700: P3; 800-1500: P4; 8002000: P5; 1000-1200: P6; 1500-2000: P7; 1500-2500: P8; 2000-2500: P9; 3000-3500: P10) y altitud (en metros) con intervalos (0-100: AO; 101-200: A1; 201-300: A2; 301-400: A3; 401-500: A4; 501-600: A5; 801-900: A8; 10011100: A10; 1101-1200: A11; 1201-1300: A12; 1301-1400:A13; 1401-1500: A14).

FAMILIA HELICINIDAE

Helicina chrysocheila Binney 1851

H. flavida Menke, 1828

H. vannatae Pilsbry, 1909

H. zephyrina Duclos, 1833

Schasicheila hidalgoana Dall, 1897

S. minuscula Pfeiffer, 1859

S. xanthia Pilsbry, 1909

FAMILIA CERESIDAE

Ceres nelsoni Dall, 1898

FAMILIA CYCLOPHORIDAE

Aperostoma mexicanum palmeri

(Bartsch y Morrison, 1942)
MX; TS2, TS3, TS5; HB, HG, HH, HI; P2, P5, P8, P9; A3, A4, A10. BMM; TS6; HI; P10; A2, A5, A8.

BTCS, BMM, BEN; TS6; HG, HH, HI; P5, P8, P10; A2, A4, A5, A8. BE, BTCS, BTP, PC; TS3, TS5, TS6; HC, HE, HF, HH, HI; P4, P7, P8, P9; A0, A1, A2, A3.

BTCS, BTP, BMM, BEN, PC; TS3, TS5, TS6; HG, HH, HI; P5, P8, P9, P10; A1, A2, A4, A5, A8, A13, A14.

BMM; TS6; HI; P9, P10; A5, A8.

BEN; TS5; HI; P9; A5.

BMM, BEN; TS5, TS6; HI; P9, P10; A8, A10.

BMM; TS6; HH; P10; A8. 
Correa-Sandoval et al. Ecología de los gastrópodos de San Luis Potosí, México

FAMILIA DIPLOMMATINIDAE

Adelopoma stolli Martens, 1890

BTP; TS6; HI; P9; A1

FAMILIA VERONICELLIDAE

Leidyula moreleti (Crosse y Fischer, 1872) BTP; TS6; HI; P9; A2.

FAMILIA CARYCHIIDAE

Carychium mexicanum Pilsbry, 1891

BMM; TS6; HI; P10; A8.

FAMILIA PUPILLIDAE

Gastrocopta contracta (Say, 1822)

G. corticaria (Say, 1816)

G. pellucida (Pfeiffer, 1841)

Pupisoma dioscoricola insigne

Pilsbry, 1920

BE, BTCS, BMM; TS6; HC, HH, HI; P4, P8, P10; A0, A1, A4, A8. BEN; TS6; HG; P5; A8.

MX, BE, BTCS, BTP, BEN, PC; TS2, TS3, TS5, TS6; HA, HB, HC, HE, HF, HG, HH, HI; P1, P2, P4, P5, P6, P7, P8, P9; A0, A1, A2, A3, A4, A10, A12, A13.

MX, BE, BTCS, BTP, BMM, BEN, PC; TS2, TS3, TS5, TS6; HB, HC, HE, HF, HG, HH, HI; P2, P4, P5, P7, P8, P9, P10; A0, A1, A2, A3, A4, $\mathrm{A} 5, \mathrm{~A} 8, \mathrm{~A} 10$.

P. minus Pilsbry, 1920

BTCS, BTP, BMM, BEN; TS5, TS6; HG, HH, HI; P2, P8, P9, P10; A1, A2, A4, A5, A8, A13.

Vertigo ovata Say, 1822

BE; TS6; HE, HI; P4, P10; A1.

FAMILIA STROBILOPSIDAE

Strobilops aenea mexicana Pilsbry, 1903

S. hubbardi Brown, 1861

BMM; TS6; HI; P10; A5, A8.

BTCS, BMM, BEN; TS6; HG, HH, HI; P5, P8, P10; A4, A8.

FAMILIA SUCCINEIDAE

Succinea luteola

MX, BE, BTCS, BEN, PC; TS3, TS5, TS6; HA, HC, HF, HG, HH; P1, P4, P5, P6, P7, P8; A0, A1, A2, A4, A11, A13.

FAMILIA DISCIDAE

Gonyodiscus victorianus (Pilsbry, 1903)

BE, BTCS, BTP, PC; TS5, TS6; HC, HH, HI; P4, P6, P8, P9; A0, A1, A2, A13.

FAMILIA PUNCTIDAE

Punctum minutissimum (Lea, 1841)

BTP, BMM, BEN; TS5, TS6; HG, HI; P5, P9, P10; A1, A5, A8, A13.

FAMILIA CHAROPIDAE

Chanomphalus pilsbryi (Baker, 1927)

BTCS, BTP, BMM, BEN, PC; TS5, TS6; HF, HG, HH, HI; P5, P7, P8, P9, P10; A1, A2, A3, A4, A5, A8.

FAMILIA ZONITIDAE

Glyphyalinia sp

BEN; TS6; HG; P5; A8, A14.

Hawaiia minuscula (Binney, 1840)

BE, BTCS, BTP, BMM, BEN, PC; TS5, TS6; HC, HE, HF, HG, HH, HI; P4, P5, P6, P7, P8, P9, P10; A0, A1, A2, A4, A5, A8, A14.

Zonitoides arboreus (Say, 1816) BMM, BEN; TS6; HG, HI; P5, P10; A5, A8.

FAMILIA SYSTROPHIIDAE

Miradiscops opal (Pilsbry, 1919)

BTCS, BTP, BMM, BEN, PC; TS5, TS6; HC, HG, HH, HI; P4, P5, P8, P9; A0, A1, A2, A5, A8. 
M. puncticipitis (Pilsbry, 1926)

FAMILIA HELICARIONIDAE

Guppya gundlachi (Pfeiffer, 1839)

G. micra Pilsbry, 1903

G. sterkiia punctum Baker, 1930

Habroconus elegantula (Pilsbry, 1919)

FAMILIA FERUSSACIIDAE

Cecilioides consobrina veracruzensis

(Crosse y Fischer, 1877)

FAMILIA SUBULINIDAE

Beckianum beckianum (Pfeiffer, 1846)

Lamellaxis gracilis (Hutton, 1834)

L. micra (Orbigny, 1835)

Leptinaria mexicana (Pfeiffer, 1866)

L. tamaulipensis Pilsbry, 1903

FAMILIA SPIRAXIDAE

Coelostele tampicoensis Pilsbry, 1906

Euglandina corneola (Binney, 1857)

E. lamyi (Fischer y Chatelet, 1903)

E. oblonga potosiana Pilsbry, 1908

E. texasiana (Pfeiffer, 1857)

Euglandina sp

Salasiella hinkleyi Pilsbry, 1919

Salasiella sp

Spiraxis sp

Streptostyla bartschii Dall, 1908

S. gracilis Pilsbry, 1907

S. jilitlana Dall, 1908

S. palmeri Dall, 1905

S. potosiana Dall, 1905

S. supracostata Pilsbry, 1909

FAMILIA SAGDIDAE

Microconus sp

Thysanophora fuscula (Adams, 1849)
BTP; TS6; HI; P9; A1.

BE, BTCS, BTP, BMM, BEN; TS5, TS6; HE, HF, HG, HH, HI; P4, P5, P7, P8, P9, P10; A1, A2, A3, A4, A5, A8, A13.

BE, BTCS, BTP, BMM, BEN, PC; TS5, TS6; HE, HF, HG, HH, HI; P4, P5, P7, P8, P9, P10; A1, A2, A3, A4, A5, A8, A13, A14. BTP, BMM, BEN; TS6; HG, HI; P5, P9, P10; A1, A5, A8, A13, A14. BTCS, BTP, BEN, PC; TS3, TS5, TS6; HG, HH, HI; P5, P7, P8, P9; A1, A2, A3, A4, A8, A13, A14.

MX, BE, BTCS, BTP, BMM, BEN, PC; TS2, TS3, TS5, TS6; HB, HC, HF, HG, HH, HI; P2, P4, P5, P7, P8, P9, P10; A0, A1, A2, A3, A4, A5, A8, A10, A13, A14.

BTP, BMM; TS5, TS6; HI; P9, P10; A1, A2, A5, A8, A14.

BTCS, BMM, PC; TS5, TS6; HC, HH, HI; P4, P6, P8, P10; A0, A1, A2, $\mathrm{A} 4, \mathrm{~A} 8$.

BTP, BMM; TS6; HI; P9, P10; A1, A8.

BTCS, BTP, BMM, BEN, PC; TS5, TS6; HF, HG, HH, HI; P5, P7, P8, P9, P10; A1, A2, A3, A4, A5, A8.

BTCS, BEN; TS6; HF, HG; P5, P7; A3.

BTCS, BMM, BEN, PC; TS5, TS6; HC, HG, HH, HI; P4, P5, P8, P10; $\mathrm{A} 0, \mathrm{~A} 2, \mathrm{~A} 3, \mathrm{~A} 5, \mathrm{~A} 8, \mathrm{~A} 13$.

BTCS, BTP, BMM, BEN, PC; TS3, TS5, TS6; HG, HH, HI; P5, P8, P9, P10; A1, A2, A4, A5.

MX; TS5; HA; P1; A12.

BTCS, BEN; TS6; HG, HH; P5, P8; A2, A13.

BTCS, PC; TS3, TS5, TS6; HC, HH; P4, P6, P8; A0, A2, A4.

BMM, BEN; TS5, TS6; HI; P9, P10; A4, A5.

BTCS, BMM, BEN, PC; TS5, TS6; HF, HG, HH, HI; P5, P7, P8, P10; A1, A2, A4, A5, A8, A13.

BTCS; TS6; HH; P8; A2.

BTCS, BMM, BEN; TS6; HG, HH, HI; P5, P8, P10; A4, A5, A13, A14.

BMM, BEN; TS5; HI; P9, P10; A4, A8.

BTCS, BMM, BEN, PC; TS3, TS5, TS6; HG, HH, HI; P5, P8, P10; A2, A4, A5, A8, A13.

BMM, BEN; TS5, TS6; HG, HI; P5, P9, P10; A4, A5, A13.

MX, BMM, BEN; TS2, TS3, TS6; HB, HG, HI; P2, P5, P10; A8, A10, A13.

BEN, TS6; HG; P5; A14.

BMM, BEN; TS5, TS6; HI; P9, P10; A4, A5, A8.

BMM; TS6; HI; P10; A8.

BE, BTCS, BTP, BMM, BEN, PC; TS5, TS6; HE, HF, HG, HH, HI; P4, P5, P7, P8, P9, P10; A1, A2, A3, A4, A5, A8, A10, A13. 
Correa-Sandoval et al. Ecología de los gastrópodos de San Luis Potosí, México

T. hornii (Gabb, 1866)

FAMILIA UROCOPTIDAE

Coelocentrum priosculpta

Thompson y Correa-Sandoval, 1994

C. tanydeira Thompson, 1968

Holospira hinkleyi Pilsbry, 1907

Microceramus mexicanus (Martens, 1897)
MX, BE, BTCS, BTP, BMM, BEN, PC; TS2, TS3, TS5, TS6; HA, HB, HC, HD, HE, HF, HG, HH, HI; P1, P2, P3, P4, P5, P6, P7, P8, P9, P10; A0, A1, A2, A3, A4, A5, A8, A10, A12, A13, A14.

BEN; TS6; HG; P5; A13.

BMM, BEN; TS5, TS6; HI; P9, P10; A4, A5, A8.

BTCS; TS6; HH; P8; A2.

MX, BTCS, BTP, BEr
P9; A1, A2, A4, A8.

FAMILIA BULIMULIDAE

Drymaeus emeus (Say, 1829)

D. multilineatus (Say, 1825)

D. sulphureus (Pfeiffer, 1857)

Orthalicus princeps (Sowerby, 1833)

Rabdotus alternatus (Say, 1830)

FAMILIA POLYGYRIDAE

Polygyra cereolus carpenteriana (Bland, 1860)

P. implicata (Martens, 1865)

P. oppilata (Morelet, 1849)

Praticolella berlandieriana (Moricand, 1833)

P. martensiana (Pilsbry, 1907)

FAMILIA XANTHONYCIDAE

Trichodiscina cordovana (Pfeiffer, 1858). BTCS, BMM, BEN; TS6; HG, HH, HI; P5, P8, P10; A5, A8.

Recibido: 3 de noviembre de 2007

Aceptado: 3 de noviembre de 2008
BTCS, BEN; TS6; HG, HH; P5, P8; A2, A4.

BTCS; TS6; HI; P8, P9, P10; A4

BTP, BMM; TS6; HI; P9, P10; A1, A5.

BTCS; TS6; HH; P8; A4.

MX, BTCS; TS5; HA, HH; P1, P6, P8; A2, A3, A12.

MX; TS5; HB; P2; A8.

BTCS, BTP, PC; TS3, TS5, TS6; HC, HF; P4, P8, P9; A0, A1, A3, A4. BE, BTCS, PC; TS3, TS5, TS6; HC, HE, HF; P4, P7; A0, A1

P4, P5, P6, P7, P8, P9; A0, A1, A2, A3, A4, A8, A13.

MX, BTCS; TS5, TS6; HB, HH; P2, P8; A2, A4, A8. 